NEW ZEALAND INSTITUTE FOR THE STUDY

OF COMPETITION AND REGULATION INC.

\title{
Carbon Subsidies and Optimal Forest Management
}

\author{
Graeme A. Guthrie \\ Victoria University of Wellington, Wellington, New Zealand
}

\author{
Dinesh K. Kumareswaran \\ New Zealand Institute for the Study of Competition and Regulation
}

December 23, 2003 


\title{
Carbon Subsidies and Optimal Forest Management*
}

\author{
Graeme A. Guthrie ${ }^{\dagger}$ \\ New Zealand Institute for the Study of Competition and Regulation \\ and Victoria University of Wellington \\ Dinesh K. Kumareswaran \\ New Zealand Institute for the Study of Competition and Regulation
}

December 23, 2003

\begin{abstract}
We consider the effect of carbon subsidies and taxes, in the form of carbon credit allocations, on forest owners' land use and harvest decisions. We introduce three possible credit allocation regimes: one where credits are allocated according to the annual flow of carbon, another where annual credits are proportional to the stock of carbon, and a third involving lump sum payments. Using a real options model with uncertain future timber prices, we examine the effect on the timing of harvest, the replanting-abandonment decision, and the value of a forest. We show that forests are less likely to be converted to alternative land uses under all three regimes, relative to the situation without any carbon credit allocation. We also show that the flows and stocks schemes lengthen optimal rotations, while lump sum allocations shorten them. Thus, the objectives of reduced deforestation and longer rotations are best met by the flows and stocks schemes. Our numerical experiments suggest that these two regimes yield very similar outcomes.
\end{abstract}

JEL classification: Q23, Q28, Q15

Keywords: Optimal forest rotation length, real options, carbon credits

\section{Introduction}

Carbon dioxide is widely believed to be one of the main greenhouse gases contributing to climate change, and governments throughout the world are actively considering ways of reducing carbon dioxide emissions. Forestry experts with the Intergovernmental Panel on Climate Change (IPCC) suggest that trees, which remove carbon dioxide from the atmosphere and store it as carbon, have the potential to sequester significant amounts of carbon. However, in the absence of carbon subsidies or taxes, forest owners consider only the cash flows from harvesting and selling timber when managing their forests. It is likely, therefore, that current forestry management practices are not optimal when the public good benefits of carbon sequestration are considered. This paper examines the ability of carbon subsidies and taxes to incentivize forest owners to increase forest land and lengthen rotations, both steps which will increase overall carbon storage and help mitigate climate change.

${ }^{*}$ We thank Lewis Evans for many very helpful comments. Kevin Counsell, Steen Videbeck, and participants at an ISCR seminar also provided useful comments. Fletcher Forests Ltd and the New Zealand Ministry of Agriculture and Forestry generously provided data.

${ }^{\dagger}$ Corresponding author: School of Economics and Finance, Victoria University of Wellington, PO Box 600, Wellington, New Zealand. Ph: 64-4-4635763. Fax: 64-4-4635566. Email: graeme.guthrie@vuw.ac.nz 
The Kyoto Protocol is one attempt by the world community to reduce the emission of greenhouse gases. Under the Protocol, forest sinks would earn tradable emission units, so-called carbon credits, in exchange for sequestering carbon, thus providing an incentive to enhance such sinks. Governments may allocate these credits to domestic forest owners or retain them as they see fit, but distribution of carbon credits to forest owners will be necessary if there are to be any incentive effects. We examine this issue in this paper, focussing on alternative carbon credit payment systems that governments may use to allocate credits to growers.

A thorough analysis of the problem needs to allow for alternative land uses, because one of the key goals of distributing carbon credits is to deter forest owners from switching to alternative land uses. Since one of the key determinants of such a change, as well as of the timing of harvest decisions, is the level of timber prices, we must also allow for volatility in future timber prices. Thus, we follow the so-called 'real options' approach to modelling optimal harvesting decisions. (See, for example, Morck, et al. (1989) and Insley (2002).) An important insight of the real options approach is that when decisions are costly to reverse and made under uncertainty, there is a value in delaying decisions. Options, such as that to harvest a forest, will only be exercised when the payoff from doing so exceeds the value of waiting. Thus, a forest will not be harvested as soon as doing so is a break-even action. Rather, the forest owner will wait, gathering more information about future timber prices, before committing to the harvest decision - and, perhaps more importantly, before deciding whether to replant the land or switch to an alternative use. When uncertainty is greater, the forest owner will delay longer.

We consider three different schemes for allocating carbon credits to forest owners. In the simplest regime, forest owners receive a lump sum payment whenever they plant a forest, and must repay the same lump sum as soon as the forest is harvested. (If a forest is replanted immediately after harvesting, the net payment is zero). The other two regimes allocate carbon credits in proportion to the change in the forest's carbon stock, called the flows scheme, and to the level of the carbon stock, called the stocks scheme, respectively. Thus, under the flows scheme the forest owner must repay the carbon credits accumulated during the life of the forest as soon as it is harvested. The forest owner is not liable to any such repayment under the stocks scheme.

The forest owner has a strong incentive not to switch to an alternative land use under the lump sum scheme, and by making the outside option less attractive this scheme lowers the value of delaying the harvest decision, therefore leading to shorter rotations than should be the case without carbon credits. Thus, while a lump sum scheme would discourage deforestation, it would encourage more rapid rotations, implying an ambiguous result for overall carbon sequestration. Despite the differences between the flows and stocks schemes, we find that they induce remarkably similar behavior from forest owners. The increased value of the forest resulting from the carbon credit cash flows discourages forest owners from exiting the industry. However, replanting is actually delayed under these regimes, despite the reduced value of waiting to determine the optimal future land use. This occurs because harvesting a forest would result in the relatively high carbon credit cash flows earned by a mature forest being replaced by the much lower carbon credit cash flows earned by a newly-planted one. Thus, the flows and stocks schemes both discourage deforestation and encourage longer rotations, both leading to enhanced carbon sequestration.

Other authors have considered the interaction between carbon sequestration and optimal rotation length. Van Kooten, et al. (1995) use a deterministic model of a forest which is always replanted upon harvesting to study the socially-optimal rotation length under different assumptions regarding carbon value, the amount of carbon released upon harvesting, etc. Romero, et al. (1998) approach the problem from a social planner's point of view and, using a deterministic model, examine the trade-off between the value of harvested timber and the value of carbon uptake for a particular family of social welfare functions. They calculate subsidies which induce forest owners to choose socially-optimal rotation lengths. Sohngen and Mendelsohn (2003) describe a deterministic general equilibrium model, and use it to derive endogenous time paths 
for the price of carbon and the price of timber. However, all of these authors use deterministic models and, with the exception of Sohngen and Mendelsohn, ignore the possibility of changing land use which is so important under any carbon credit regime.

In the next section we describe the carbon credit allocation schemes which we will consider in this paper. We describe our model in Section 3, and calibrate it to New Zealand data in Section 4. The optimal forest management policy is analyzed in Section 5. The next section discusses the implications of our findings for policymakers, and Section 7 offers some concluding remarks.

\section{Three alternative payment systems}

Under the Kyoto Protocol (UNFCCC, 1997), credits earned for sequestering carbon dioxide would be allocated to the governments that have ratified the international treaty. Forest sinks may also be the point of obligation for the release of emissions relating to harvest known as Kyoto obligations. The governments may allocate these credits and Kyoto obligations to domestic forest owners or retain them as they see fit. Since there is still much uncertainty over the mechanics of how credits will be allocated to growers, in this section we introduce three alternative schemes for allocating credits, and discuss the merits of each. We assume throughout that the government is willing to pass on both carbon credits and Kyoto obligations to growers.

Lump sum regime. Afforestation, reforestation, and deforestation are infrequent and costlyto-reverse events that greatly alter the carbon density of a unit of land. ${ }^{1}$ The Kyoto Protocol, which is concerned with land-use change, rewards afforestation and reforestation and penalizes deforestation when allocating credits to the Parties. The lump sum regime is a means by which carbon credit allocations to forest owners can mirror allocations to the Parties and reflect these one-off changes to the carbon landscape. It entitles forest owners to a one-off lump sum payment for engaging in afforestation or reforestation, paid at the commencement of the first rotation. Growers retain these credits as long as replanting occurs immediately after harvest; if the forest is not replanted, deforestation has occurred, the owner incurs a Kyoto obligation, and the lump sum allocation must be repaid in full.

Flows regime. Under this scheme, which is the one modelled by van Kooten, et al. (1995), the carbon credit cash flows are a function of the annual change in the forest carbon stock. Credit payments reflect the flow of carbon between land and the atmosphere via the carbon cycle, so a net increase in the carbon stock over a year means the sink has removed carbon from the atmosphere and the owner is paid credits for the carbon. Similarly, a fall in the carbon stock suggests carbon has been released into the atmosphere, and the owner surrenders the associated credits. Therefore, the forest attracts credits as long as it is growing. The moment it is harvested, it is assumed that all the sequestered carbon is released back into the atmosphere so the owner incurs a Kyoto obligation and all the credits earned throughout the life of the forest must be repaid. ${ }^{2}$ Upon replanting, the owner starts to re-accumulate credits. If the owner

\footnotetext{
${ }^{1}$ Afforestation is "the direct human-induced conversion of land that has not been forested for a period of at least 50 years to forested land through planting, seeding, and/or the human-induced promotion of natural seed sources", reforestation is "the direct human-induced conversion of non-forested land to forested land through planting, seeding, and/or the human-induced promotion of natural seed sources, on land that was forested but that has been converted to non-forested land", and deforestation is "the direct human-induced conversion of forested land to non-forested land" (UNFCCC, 2001).

${ }^{2}$ In practice, harvest does not result in the total removal of all biomass. Some above-ground biomass (ground litter, fallen branches), as well as some below-ground biomass (semi-decomposed litter, roots) will remain behind even after the removal of the trees. Over time this matter will break down completely but as this occurs more will accumulate from subsequent rotations maintaining a roughly stable minimum carbon stock on the land. However for simplicity, and in keeping with IPCC assumptions that the biomass from the forest breaks down immediately after harvest, we assume that the carbon stock on the land goes to zero upon harvest.
} 
decides not to replant, then the credits have been surrendered forever.

Stocks regime. Forest sinks remove carbon from the atmosphere during periods of growth, but eventually release carbon back into the atmosphere following harvest, hence providing only temporary benefits. Nevertheless, even temporary carbon capture generates a positive environmental externality during the period of storage. The stocks scheme, which is the one modelled by Sohngen and Mendelsohn (2003), rewards this temporary storage by giving forest owners credit not only for the quantity of carbon captured, but also for the duration of capture, effectively treating carbon credits as rent payments for the storage of carbon. Under this regime, credits are allocated to the owner annually in proportion to the size of the total carbon stock, which grows cumulatively through time. As long as carbon is being held the forest receives rent in the form of carbon credits. ${ }^{3}$

\section{The model}

In this paper we value a representative forest receiving carbon credit payments, allowing us to examine the effect of these payments on the optimal behavior of forest owners. When the forest is $t$ years old, the volume of timber (per hectare) equals $V(t)$, for some (deterministic) function $V$. We assume the price of timber, $P$, evolves according to the Itô process

$$
d P=\mu(P) d t+\sigma P d z
$$

where $\mu(P)$ is a general drift term that is a function of timber prices, $\sigma$ is a positive constant, and $z$ is a Wiener process. We also assume that timber stocks have a convenience yield $\kappa P$, for some constant $\kappa$, so that the 'risk-neutral' process for the price of timber is ${ }^{4}$

$$
d P=(r-\kappa) P d t+\sigma P d z,
$$

where $r$ is the riskless interest rate. Over each increment of time, $d t$, the owner of a forest which is $t$ years old receives an after-tax cash flow of $\psi(t) d t$, comprising carbon credit revenues (calculated according to one of the three regimes introduced in Section 2) less non-harvest related costs. $^{5}$ The appropriate values for the carbon credit cash flows under each of the three schemes, denoted by $Q(t)$, are summarized in Table 1 .

At each point in time, the forest owner must take one of three possible actions: decide to harvest the forest and convert the land to some alternative use; decide to harvest the forest and replant the land; or delay the harvest decision. The value of a forest which is $t$ years old is

$$
F(P, t)=\max \left\{\pi_{t}^{a}, \pi_{t}^{r}, \pi_{t}^{d}\right\},
$$

where $\pi_{t}^{a}, \pi_{t}^{r}$, and $\pi_{t}^{d}$ denote the respective payoffs to abandoning, replanting, and delaying.

Since the owner requires a lead-time of $\bar{T}$ years in order to mobilize for harvest, the decision to harvest is always made $\bar{T}$ years in advance. When this decision is made, fixed (after-tax) pre-harvest costs $\delta$ are immediately incurred. The abandonment payoff is therefore ${ }^{6}$

$$
\pi_{t}^{a}=-\delta+\int_{0}^{\bar{T}} e^{-r s} \psi(t+s) d s+e^{-r \bar{T}} E\left[\left(1-T_{c}\right)\left(P_{t+\bar{T}}-h\right) V(t+\bar{T})+\hat{Q}(t+\bar{T})+S\right],
$$

\footnotetext{
${ }^{3}$ The absence of Kyoto obligations under this regime makes it an ideal means to allocate credits by the Clean Development Mechanism to non-Annex I countries, who face no obligations under the Protocol and therefore cannot be penalized for harvest-related emissions.

${ }^{4}$ For a detailed discussion of the role that 'risk-neutral' processes play in valuation, see Dixit and Pindyck (1994, Chapter 4).

${ }^{5}$ In practice these cash flows arise discretely through time (annually or as one-off amounts), but as we value the forest in continuous time we assume a continuous cash flow stream. See Appendix A for a summary of the forest's cash outflows.

${ }^{6}$ The expected value in this expression is calculated using the 'risk-neutral' process for the price of timber, ensuring that forest values are arbitrage-free. The same is true of all other expected values in this paper.
} 
Table 1: Carbon credit cash flows under the three schemes

\begin{tabular}{|c|c|c|}
\hline Regime & $Q(t)$ & $\hat{Q}(t)$ \\
\hline Lump sum & $\begin{array}{ll}x^{l} & \text { if } t=0 \\
0 & \text { if } t>0 .\end{array}$ & $-x^{l}$ \\
\hline Flows & $x^{f} V^{\prime}(t)$ & $-x^{f}(V(t)-V(0))$ \\
\hline Stocks & $x^{s} V(t)$ & 0 \\
\hline
\end{tabular}

Notes: The entries in the table describe the carbon credit regimes introduced in Section 2. The precise levels of the cash flows depend on regime-specific constant parameters $x^{l}, x^{f}$, and $x^{s}$, reflecting the quantity of credits allocated and the price of carbon credits (assumed constant over time). The column labelled $Q(t)$ shows the carbon credit cash flow received by the owner of a $t$-year old forest while it is growing, and the column labelled $\hat{Q}(t)$ shows the carbon credit cash flow when the corresponding forest is harvested.

where $T_{c}$ is the corporate tax rate, $h$ represents harvest costs (per unit of timber), $V(t+\bar{T})$ is the volume of timber extracted at the harvest date, $\hat{Q}(t+\bar{T})$ denotes the after-tax carbon credit cash flows when harvest occurs (specified in Table 1), and $S$ is some fixed scrap value received by the owner in the event that forestry is abandoned in favor of an alternative land-use. The first term in (3) represents the costs incurred at the time the harvesting decision is made, the second (integral) term gives the value of all cash flows received until harvest occurs, and the third term is the present value of the harvest-date cash flows. The payoff from harvesting, and then replanting, the forest is similar, with the scrap value $S$ being replaced by the value of a newly-planted forest:

$$
\begin{aligned}
\pi_{t}^{r}= & -\delta+\int_{0}^{\bar{T}} e^{-r s} \psi(t+s) d s \\
& +e^{-r \bar{T}} E\left[\left(1-T_{c}\right)\left(P_{t+\bar{T}}-h\right) V(t+\bar{T})+\hat{Q}(t+\bar{T})+F\left(P_{t+\bar{T}}, 0\right)\right] .
\end{aligned}
$$

Finally, if harvest does not occur during the next short interval of length $d t$, then the payoff is

$$
\pi_{t}^{d}=\psi(t) d t+e^{-r d t} E[F(P+d P, t+d t)],
$$

comprising the sum of the cash flow received during this interval and the present value of the (unharvested) forest at the end of the interval. Using Itô's Lemma, this equals

$$
\pi_{t}^{d}=\psi(t) d t+F(P, t)+\left(\frac{\partial F}{\partial t}+\frac{1}{2} \sigma^{2} P^{2} \frac{\partial^{2} F}{\partial P^{2}}+(r-\kappa) P \frac{\partial F}{\partial P}-r F\right) d t
$$

In the region where delay is optimal, $F(P, t)=\pi_{t}^{d}$, so that $F$ must satisfy the partial differential equation

$$
0=\frac{\partial F}{\partial t}+\frac{1}{2} \sigma^{2} P^{2} \frac{\partial^{2} F}{\partial P^{2}}+(r-\kappa) P \frac{\partial F}{\partial P}-r F+\psi(t) .
$$

The optimal harvest policy is described by two thresholds, $P^{a}(t)$ and $P^{r}(t)$, which are functions of the age of the forest. If the timber price is less than $P^{a}(t)$ when an established forest is $t$ years old, then the forest owner should immediately begin the harvest process and, when harvest is complete, convert the land to an alternative land use; if the timber price exceeds $P^{r}(t)$ when an established forest is $t$ years old, then the forest owner should immediately begin the harvest process and, when harvest is complete, replant trees on the land; if the timber price lies between these thresholds, then the forest owner should delay the harvest decision. Thus $F$ equals $\pi_{t}^{a}$ when $P \leq P^{a}(t)$, it equals $\pi_{t}^{r}$ when $P \geq P^{r}(t)$, and it satisfies (5) when $P^{a}(t)<P<P^{r}(t)$. 
Table 2: Discrete-time estimates of price process

\begin{tabular}{ccc}
\hline$\hat{\alpha}$ & $\hat{\beta}$ & $\hat{\phi}$ \\
\hline 0.83 & 0.83 & 0.10 \\
$(0.25)$ & $(0.05)$ & \\
\hline
\end{tabular}

Notes: The table reports OLS estimates of the process in (7), with standard errors given in parentheses. $\hat{\phi}$ is the standard error of the regression.

Table 3: Parameter summary

\begin{tabular}{cccc}
\hline Parameter & Value & Parameter & Value \\
\hline$\hat{a}$ & 0.73 & $\hat{\gamma}_{3}$ & -0.01512 \\
$\hat{b}$ & 4.97 & $r$ & 0.05 \\
$\hat{\sigma}$ & 0.25 & $\kappa$ & 0.075 \\
$\hat{\gamma}_{1}$ & -2.676 & $T_{c}$ & 0.33 \\
$\hat{\gamma}_{2}$ & 1.224 & $S$ & 1087.15 \\
\hline
\end{tabular}

Notes: $\hat{a}, \hat{b}$, and $\hat{\sigma}$ are our estimates of the parameters appearing in (6), the timber price process. $\hat{\gamma}_{1}, \hat{\gamma}_{1}$, and $\hat{\gamma}_{1}$ determine the process (8) which describes the volume of timber in the forest. $r$ is the continuously-compounded riskless interest rate, $\kappa$ is the convenience yield, $T_{c}$ is the corporate tax rate, and $S$ is the value of the land (in dollars per hectare) in its next most valuable use.

\section{Calibration of model parameters}

The historical timber price data used in this paper consists of quarterly observations on New Zealand A-Grade Pinus radiata logs (1973:1-2001:3). All prices are measured in New Zealand dollars per Japanese Agricultural Standard cubic meter, priced free-on-board, and are adjusted from nominal to real December 1999 levels. Making a common assumption for commodity prices, and consistent with the process in (1), we assume that the natural logarithm of timber prices, $p=\log P$, evolves according to the Ornstein-Uhlenbeck process

$$
d p=a(b-p) d t+\sigma d z,
$$

where $a$ is the rate of mean-reversion and $b$ is the long-run level of $\log P$. This process may be modelled in discrete-time using the AR(1) process

$$
p_{t}=\alpha+\beta p_{t-1}+u_{t}, \quad u_{t} \sim N\left(0, \phi^{2}\right) .
$$

The OLS estimates of (7) are presented in Table 2. From these estimates, it is possible to estimate the parameters in (6) via

$$
\hat{a}=\frac{-\log \hat{\beta}}{\Delta t}, \quad \hat{b}=\frac{\hat{\alpha}}{1-\hat{\beta}}, \quad \hat{\sigma}=\hat{\phi} \sqrt{\frac{-2 \log \hat{\beta}}{\left(1-\hat{\beta}^{2}\right) \Delta t}},
$$

where $\Delta t=1 / 4 .^{7}$ These estimates are reported in Table 3 .

The wood volume data used in this paper was generated using the forestry software package STANDPAK developed by the New Zealand Forest Research Institute (Whiteside, 1990). STANDPAK was used to simulate the volume of wood in a representative single-hectare Pinus radiata forest with a site index of 29 meters. ${ }^{8}$ We use OLS to fit the simple cubic function

$$
V(t)=\gamma_{1} t+\gamma_{2} t^{2}+\gamma_{3} t^{3}
$$

\footnotetext{
${ }^{7}$ See, for instance, Dixit and Pindyck (1994, Chapter 3).

${ }^{8}$ The site index is the mean top height at age 20 of all trees in a stand, and is used as a measure of the productivity of a unit of land.
} 
Figure 1: Optimal forest management

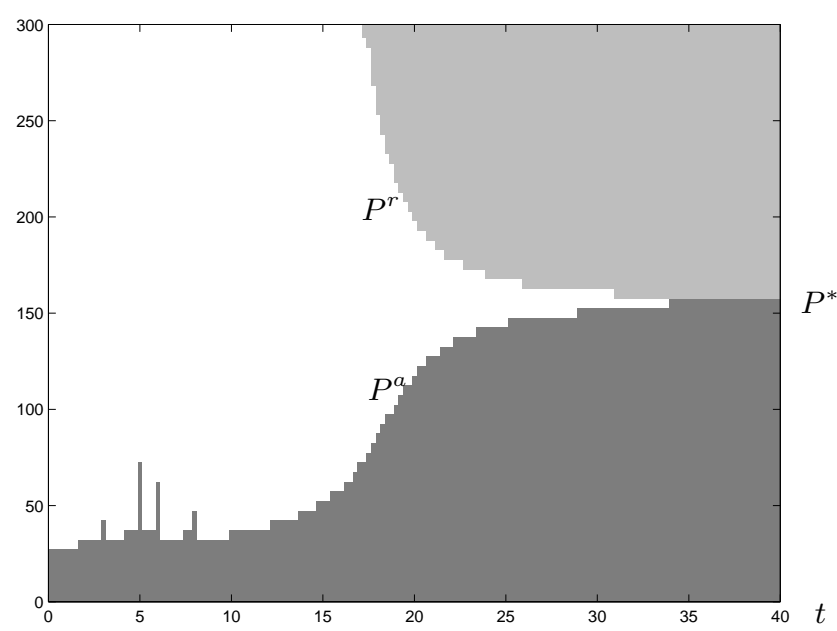

Notes: This figure represents the replanting and abandonment decision thresholds for the owner of a representative $P$. radiata forest who faces uncertainty in timber prices. Within the lightly shaded region it is optimal to harvest and then immediately replant. Within the darkly shaded region it is optimal to harvest and then abandon. In the clear region it is optimal to defer harvest. At any point in time, $P^{r}$ is the minimum price that would induce replanting and $P^{a}$ is the maximum price that would induce abandonment. $P^{*}$ is the trigger price required to persuade the owner to switch from a replanting to an abandonment decision.

to the wood flow data. The parameter estimates are presented in Table 3.

The project scrap value, $S$, is the average freehold farm sale price per hectare of grazing farmland in New Zealand for 2001 deflated to real December 1999 levels. ${ }^{9}$ This price is marketdetermined, and so captures the value to forest owners of switching to an alternative land-use. All the model parameters relevant to the numerical implementation of our model are summarized in Table 3.

In the absence of a closed-form solution to the option pricing problem, we numerically approximate a solution using the method described in Appendix B.

\section{Optimal forest management}

Figure 1 shows the replanting and abandonment thresholds for the owner of a representative plantation of Pinus radiata for the parameters specified above. Forest age is represented on the horizontal axis while the price of timber is represented on the vertical one. It is optimal to delay the harvest decision as long as the timber price path, which evolves through time according to (1), remains in the clear region in between the two thresholds. However, whenever the price path crosses either threshold, the owner should initiate the harvest process. The lightly shaded area shows where it is optimal to harvest and then immediately replant the land. The darkly shaded area shows where it is optimal to harvest and abandon forestry by converting to an alternative land-use.

The forest owner should optimally delay harvest in some situations, even when harvesting and then replanting the land (or harvesting and then converting to an alternative land use) would have a positive payoff, and our results include this behavior. The reason why such behavior can be optimal is that there is a value in waiting to gather more information. Both replanting the land and converting it to an alternative use incur sunk costs, and decision-makers wish to avoid having to reverse decisions which incur such costs. For example, forest owners want to avoid

\footnotetext{
${ }^{9}$ Source: New Zealand Ministry of Agriculture and Forestry, 2002, Table 17, p. 106.
} 
Table 4: Carbon credits and management of a representative $P$. radiata forest

\begin{tabular}{ccccccccr}
\hline$x$ & $P^{a}$ & $P^{r}$ & $P^{*}$ & $F(P ; 0)$ & $F(P ; 10)$ & $F(P ; 20)$ & $F^{W}(P ; 0)$ & $F^{Q}(P ; 0)$ \\
\hline 0 & 60 & 155 & 100 & 3427.73 & 12201.79 & 27549.03 & 3427.73 & 0.00 \\
500 & 55 & 150 & 95 & 3754.62 & 11988.93 & 27366.04 & 3423.26 & 331.36 \\
1000 & 50 & 145 & 90 & 4089.82 & 11785.76 & 27200.54 & 3409.56 & 680.26 \\
1500 & 40 & 140 & 80 & 4433.83 & 11592.96 & 27046.13 & 3388.67 & 1045.16 \\
\hline \multicolumn{8}{c}{ Flows scheme } \\
0 & 60 & 155 & 100 & 3427.73 & 12201.79 & 27549.03 & 3427.73 & 0.00 \\
1.5 & 55 & 160 & 100 & 3497.15 & 12210.74 & 27171.02 & 3426.30 & 70.85 \\
3.0 & 50 & 160 & 95 & 3569.53 & 12224.35 & 26797.88 & 3421.54 & 147.99 \\
4.5 & 40 & 165 & 95 & 3651.53 & 12249.66 & 26434.70 & 3296.96 & 354.58 \\
\hline \multicolumn{8}{c}{ Stocks scheme } \\
0 & 60 & 155 & 100 & 3427.73 & 12201.79 & 27549.03 & 3427.73 & 0.00 \\
0.075 & 55 & 160 & 100 & 3497.21 & 12331.57 & 27643.89 & 3426.31 & 70.90 \\
0.150 & 50 & 160 & 95 & 3569.64 & 12466.01 & 27743.61 & 3421.54 & 148.10 \\
0.225 & 40 & 165 & 95 & 3651.68 & 12612.14 & 27853.29 & 3297.08 & 354.60 \\
\hline
\end{tabular}

Notes: For each of the indicated carbon credit allocation regimes, columns $2-4$ of the table summarize the optimal forest management policy, with $P^{a}$ and $P^{r}$ giving the abandonment and replanting thresholds, respectively, for a forest that is 20 years old. $P^{*}$ gives the limiting value of these two thresholds as the forest's age increases without bound. Columns 5-7 report the values of forests that are 0,10 , and 20 years old, respectively. These forest values are evaluated at $P=145$, which is approximately equal to the long-run timber price from our estimate of the timber price process (6). Columns 8-9 decompose the value of a new forest into the value of the timber operations, $F^{W}$, and of the carbon credit cash flows, $F^{Q}$, again evaluated at $P=145$.

having to pull seedlings from the ground because a sudden drop in timber prices has made land conversion the optimal strategy. The risk of such events occurring can be reduced by waiting past the break-even point, to a time where prices are so high (respectively, so low) that the decision to replant (respectively, convert to an alternative land use) can be made with confidence. As we now discuss, the allocation of carbon credits can significantly alter the trade-offs facing forest owners when they make their harvest decisions.

To better understand the impact of carbon credits on the thresholds in Figure 1, we perform comparative statics analysis by varying the carbon credit payment under each scheme. The results are presented in Table 4. Under the lump sum scheme, more valuable credit allocations lead to a downward shift in both thresholds (both $P^{r}$ and $P^{a}$ fall). That is, abandonment becomes less likely, and replanting will occur sooner (even than without carbon credits). The reduced likelihood of abandonment is easily explained by the lump sum 'tax' which forest owners must pay when they exit the industry. Further, by making replanting more attractive relative to abandonment, allocating lump sum carbon credits lowers the value of waiting for more information about the optimal future land use. This, in turn, leads to earlier harvesting when replanting is optimal. Thus, while the disincentive to deforest will lead to greater carbon sequestration, the shorter rotation length will have the opposite effect. (We will return to this issue in the following section when we discuss the policy implications of the three allocation regimes.)

In contrast, under both the stocks and flows regime, increases in the value of carbon credit payments shift the replanting threshold upwards and the abandonment threshold downwards, so that rotations are longer when carbon credit payments are more valuable. The increased value of the forest resulting from the carbon credit cash flows explains the lower abandonment threshold and why, as in the lump sum case, the value of delaying the harvest decision is reduced. But now this is more than offset by the fact that, under these two schemes, carbon credit cash flows are generally larger later rather than earlier in the rotation, encouraging owners to delay harvesting in order to preserve these high cash flows.

The forest yields value to the grower in two ways: cash flows from carbon credit allocations and from saleable timber upon harvest. We can decompose the value of the forest into the 
corresponding components, $F=F^{Q}+F^{W}$, where $F^{Q}$ denotes the present value of the forest attributable to carbon credit revenues and $F^{W}$ is the present value of future cash flows from saleable timber. $F^{W}$ is easily calculated by valuing a hypothetical forest which does not receive carbon credits, yet is managed according to the replanting and abandonment thresholds for a forest which does receive carbon credits. Owners of this hypothetical forest only hold claims to timber but behave in an identical manner to owners of the forest, who hold claims to both timber and carbon credits. The two present value components of the forest under each carbon credit scheme are reported in the final two columns of Table 4, which decompose the value of a new forest into the value of the timber operations, $F^{W}$, and of the carbon credit cash flows, $F^{Q}$, again evaluated at $P=145$. This shows that the value of the saleable timber in the forest is decreasing in $x$, illustrating that while carbon credit payments increase the value of a newly-planted forest, they actually lead to sub-optimal wood production. The more valuable the payments, the greater the distortion. ${ }^{10}$ Moreover, the degree of distortion differs across the regimes. From Table 4, setting $x^{f}=4.5$ in the flows scheme results in future carbon credit revenues with present value of $\$ 354.58$, but raises the value of the forest by just $\$ 223.80$; in effect, forest owners receive just 63 cents in the dollar. For the stocks scheme with $x^{s}=0.225$, the corresponding figures are $\$ 354.60$ and $\$ 223.95$, or 63 cents in the dollar; for the lump sum scheme with $x^{l}=1500$, they are $\$ 1045.16$ and $\$ 1006.10$, or 96 cents in the dollar. The distortion caused by carbon credit payments has implications for the allocation mechanism which governments should choose. This and other policy implications are discussed in the next section.

\section{$6 \quad$ Policy implications}

In order to increase carbon sequestration, policymakers must encourage forest owners to lengthen rotations, and discourage them from converting forest land to alternative uses. That is, policymakers desire a low abandonment threshold and a high replanting one, and trade these off against the cost of carbon credits allocated to forest owners. Formally, we assume that the policymaker maximizes $U\left(F^{Q}, P^{a}, P^{r}\right)$, where utility $U$ is a decreasing function of $F^{Q}$ (defined in the previous section and interpreted here as the present value of the cost of allocating credits to growers) and $P^{a}$ (the maximum price required to induce forest owners to harvest and immediately abandon), and an increasing function of $P^{r}$ (the minimum price required to induce forest owners to harvest and immediately replant). ${ }^{11}$

The policymaker selects a carbon credit regime and chooses $x$. Forest owners then select their optimal harvest and replanting responses, given the size of the payments, thus determining $F^{Q}, P^{a}$, and $P^{r}$. This maps out the set of all possible outcomes for the social planner, who will then choose the regime and value of $x$ that maximizes $U\left(F^{Q}, P^{a}, P^{r}\right)$. The bottom two curves in Figure 2 plot the tradeoff between $F^{Q}$ and $P^{a}$ for each carbon credit scheme, while the top two curves plot the corresponding trade-off between $F^{Q}$ and $P^{r}$. The solid curves represent the lump sum scheme, and the dotted curves the stocks scheme. The flows scheme gives curves that are almost indistinguishable from the stocks scheme's. Figure 2 shows that any lump sum scheme is dominated by some stocks scheme - the stocks scheme with the same cost (that is, the same value of $F^{Q}$ ), induces less deforestation (that is, a lower value of $P^{a}$ ), and longer optimal rotations (that is, a higher value of $\left.P^{r}\right) .{ }^{12}$

\footnotetext{
${ }^{10}$ This is consistent with the findings of Hartman (1976) and Berck (1980) that failure to include the non-market and indirect financial benefits of a forest in the valuation problem may result in inefficient managerial decisions.

${ }^{11}$ We use the harvest thresholds for a 20 -year old forest, and measure the cost $F^{Q}$ of carbon credit allocations at $P=145$ and $t=0$. Our ranking of the three regimes is the same for a variety of different values of forest ages and timber prices.

${ }^{12}$ One explanation for the poor performance of the lump sum scheme is our assumption that credits are allocated at $t=0$. Since the behavior of forest owners depends on the exit tax and not on the initial payment, and the probability of abandonment is very low in the early years of the rotation, delaying the initial payment would have little effect on $P^{a}$ and $P^{r}$. However, such a delay would reduce the (present value of the) cost of the allocation, and shift the corresponding curves in Figure 2 to the left.
} 
Figure 2: Trade-off faced by the policymaker

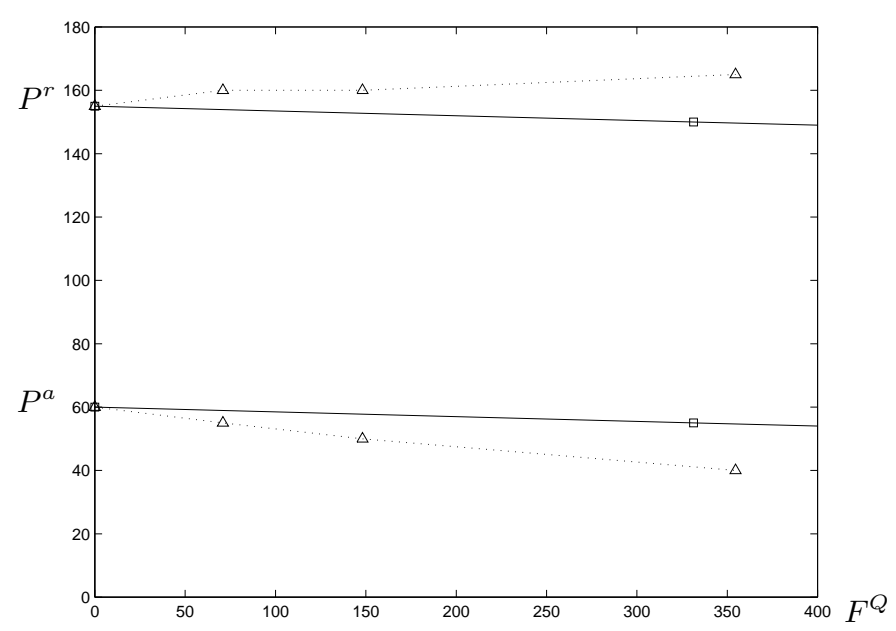

Notes: The bottom two curves plot the tradeoff between $F^{Q}$ (the horizontal axis) and $P^{a}$ (the vertical axis) for each carbon credit scheme, while the top two curves plot the corresponding tradeoff between $F^{Q}$ and $P^{r}$. $P^{a}$ and $P^{r}$, both evaluated at $t=20$, are the maximum price at which forestry is abandoned and minimum price at which the forest will be harvested and replanted, respectively. $F^{Q}$ is the cost (in present value terms) to the government of making carbon credit payments to forest owners. The solid curves represent the lump sum scheme, and the dotted curves the stocks scheme. The flows scheme gives curves that are almost indistinguishable from the stocks scheme's. Since the policymaker's objective function is decreasing in $P^{a}$ and increasing in $P^{r}$, the flows and stocks schemes are therefore preferred to the lump sum one.

A key consideration when assessing these schemes is the informational requirements for implementation to occur. For instance, both the flows and stocks schemes require annual measures of the carbon stock in order to be implemented. The cost of such frequent forest inventories could quickly overwhelm the economic benefits of the credits themselves. In contrast, the need for only one-off inventories under the lump sum regime is advantageous as it would significantly lower monitoring and compliance costs. However, under Articles 3, 5, 7, and 8 of the Protocol, Annex I Parties are required to maintain annual inventories of anthropogenic emissions by sources and removals by sinks of greenhouse gases and report these in the annual National Communications under the UNFCCC. Given these international reporting requirements, frequent stock-taking may be unavoidable. The economies of scale associated with timing carbon credit payments to coincide with these annual updates to the national inventories could yet make the flows and stocks schemes feasible.

\section{Concluding remarks}

This paper has examined how three alternative schemes for allocating carbon credits affect the harvesting and future investment decisions of forest owners. We found that the simplest scheme, in which forest owners receive a lump sum payment whenever they plant a forest, and must repay the same lump sum as soon as the forest is harvested, gives the owner a strong incentive not to switch to an alternative land use. However, by making the outside option less attractive, this lowers the value of delaying the harvest decision, and therefore leads to shorter rotations than should be the case without carbon credits. Thus, while a lump sum scheme would discourage deforestation, it would encourage more rapid rotations, implying an ambiguous result for overall carbon sequestration.

The other two regimes we considered allocate carbon credits in proportion to the change in the forest's carbon stock, called the flows scheme, or to the level of the carbon stock, called the 
stocks scheme. Despite the differences between the flows and stocks schemes, we found that they induce remarkably similar behavior from forest owners. The payment of carbon credits to forest owners raises the value of using land for forestry, thus discouraging forest owners from exiting the industry. However, unlike the lump sum scheme, replanting is delayed under these regimes. This occurs because harvesting a forest would result in the relatively high carbon credit cash flows earned by a mature forest being replaced by the much lower carbon credit cash flows earned by a newly-planted one. Thus, the flows and stocks schemes both discourage deforestation and encourage longer rotations, both leading to enhanced carbon sequestration.

In order to make the model tractable, we needed some simplifying assumptions. For example, the only source of volatility in our model is fluctuations in the price of timber. A more realistic model would allow for stochastic timber growth rates and, perhaps more importantly, a stochastic price of carbon credits. While both features could be incorporated in a model of the type used in this paper, they would significantly increase the complexity of the model and make finding a numerical approximation to the forest value much more difficult. ${ }^{13}$

Finally, our model is a partial equilibrium one. If a carbon credit allocation regime is put in place, the prices of timber and carbon credits are likely to be affected. For example, Sohngen and Mendelsohn (2003) argue that timber prices will rise initially (as longer rotations reduce the supply of timber), but will eventually fall (as more forests are planted). The difficult problem of incorporating such behavior into a real options model is worthy of future research.

\section{References}

Berck, Peter (1981). "Optimal management of renewable resources with growing demand and stock externalities," Journal of Environmental Economics and Management 8, 105-117.

Dixit, Avinash K., and Robert S. Pindyck (1994). Investment Under Uncertainty, Princeton University Press: New Jersey.

Hartman, R. (1976). "The harvesting decision when a standing forest has value," Economic Inquiry $14,52-58$.

Insley, Margaret (2002). "A real options approach to the valuation of a forestry investment," Journal of Environmental Economics and Management 44(3), 471-492.

van Kooten, G. Cornelis, Clark S. Binkley, and Gregg Delcourt (1995). "Effect of carbon taxes and subsidies on optimal forest rotation age and supply of carbon services," American Journal of Agricultural Economics 77(2), 365-374.

Morck. R., Schwartz, E., and D. Stangeland (1989). "The valuation of forestry resources under stochastic prices and inventories," Journal of Financial and Quantitative Analysis 24(2), $473-487$.

New Zealand Ministry of Agriculture and Forestry (2002). "Situation and outlook for New Zealand agriculture and forestry," Wellington, New Zealand.

Romero, C., V. Ros, and L. Daz-Balteiro (1998). "Optimal forest rotation age when carbon captured is considered: Theory and applications," Journal of the Operational Research Society $49(2), 121-131$.

Sohngen, Brent, and Robert Mendelsohn (2003). "An optimal control model of forest carbon sequestration," American Journal of Agricultural Economics 85(2), 448-457.

Statistics New Zealand (2001). Hot Off The Press, Producer Price Index, December.

\footnotetext{
${ }^{13}$ However, our model could easily be extended to incorporate trends in carbon credit prices, alternative timber growth rates, and so on. This would go some way towards exploring the impact of changes in these variables.
} 
Table 5: Pre-tax real cash outflows of a representative $P$. radiata forest

\begin{tabular}{lrc}
\hline Description & Amount & Year \\
\hline \multicolumn{1}{c}{ One-off non-harvest costs } \\
Land preparation, fencing, track establishment & 336.09 & 0 \\
Planting, fertilizing, releasing & 892.15 & 0 \\
Spraying and mapping & 36.82 & 3 \\
Pruning & 640.08 & 5 \\
& 519.24 & 7 \\
Waste thinning & 205.81 & 6 \\
& 278.50 & 9 \\
Mid-rotation inventory & 25.49 & 13 \\
\hline \multicolumn{3}{c}{ Ongoing costs } \\
Insurance, rates, maintenance, weed control, \\
fire control, road maintenance, forest health \\
survey, project management \\
\multicolumn{2}{c}{ Harvest related costs } \\
Pre-harvest inventory \\
Road preparation \\
Logging ${ }^{\ddagger}$
\end{tabular}

Notes: All costs are deflated from nominal to real December 1999 levels using a Producer Price Index for forestry and logging (see Statistics New Zealand, 2001). All costs are measured in $\$ /$ ha except those indicated with $\ddagger$ which are in $\$ / \mathrm{m}^{3}$. Data is provided by the New Zealand Ministry of Agriculture and Forestry.

UNFCCC (1997). The Kyoto Protocol to the United Nations Framework Convention on Climate Change, COP 3: Kyoto.

UNFCCC (2001). Annex to Draft Decision -/CP.6, Land-Use Change and Forestry, Section A, 6 th Session of the Conference of the Parties, Part 2, Bonn.

Whiteside, I. D. (1990). "The STANDPAK stand modeling system for radiata pine", in James, R. N., and L. Tarlton (eds.), New Approaches to Spacing and Thinning in Plantation Forestry, Proceedings of IUFRO symposium held at the Forest Research Institute, Rotorua, New Zealand, April 1989.

\section{Appendices}

\section{A Harvest and non-harvest related costs}

Our model incorporates both harvest and non-harvest related costs. The values we use, based on data provided by the New Zealand Ministry of Agriculture and Forestry, are reported in Table 5.

\section{B Numerical solution}

In the absence of a closed-form solution to the option-pricing problem, we value the forest using the explicit finite difference method. We use a series of grids, each representing a different rotation, each with nodes labelled $\left(P_{i}, t_{j}\right)$, where $P_{i}=i \Delta P$ and $t_{j}=(j-1) \Delta t-\bar{T}$. We set $\Delta P=5$ and $\Delta t=0.0025$, and include 60 steps in the $P$-direction and 16401 steps in 
the $t$-direction. ${ }^{14}$ Harvest can only occur $\bar{T}=1$ year after the harvest decision is made. Let $F_{i, j}^{k}=F\left(P_{i}, t_{j} ; k\right)$, which is the value of the forest at node $\left(P_{i}, t_{j}\right)$ in rotation $k$.

The finite difference equation corresponding to equation (5) can be written

$$
\begin{aligned}
F_{i, j}^{k}= & \frac{\Delta t}{2}\left(\sigma^{2} i^{2}-(r-\kappa) i\right) F_{i-1, j+1}^{k}+\left(1-\Delta t\left(\sigma^{2} i^{2}+r\right)\right) F_{i, j+1}^{k} \\
& +\frac{\Delta t}{2}\left(\sigma^{2} i^{2}+(r-\kappa) i\right) F_{i+1, j+1}^{k}+\psi_{j+1},
\end{aligned}
$$

where $\psi_{j} \equiv \psi\left(t_{j}\right)$. Therefore the payoff from delaying the harvest decision at node $\left(P_{i}, t_{j} ; k\right)$ is

$$
\begin{aligned}
\pi_{i, j}^{d, k}= & \frac{\Delta t}{2}\left(\sigma^{2} i^{2}-(r-\kappa) i\right) F_{i-1, j+1}^{k}+\left(1-\Delta t\left(\sigma^{2} i^{2}+r\right)\right) F_{i, j+1}^{k} \\
& +\frac{\Delta t}{2}\left(\sigma^{2} i^{2}+(r-\kappa) i\right) F_{i+1, j+1}^{k}+\psi_{j+1} .
\end{aligned}
$$

Since, under the risk-neutral process for the timber price, $E_{t}\left[P_{t+\bar{T}}\right]=P_{t} e^{(r-\kappa) \bar{T}}$, it follows that the abandonment payoff in equation (3) can be rewritten as

$$
\pi^{a}=-\delta+\int_{0}^{\bar{T}} e^{-r s} \psi(t+s) d s+e^{-r \bar{T}}\left(\left(1-T_{c}\right)\left(P e^{(r-\kappa) \bar{T}}-h\right) V(t+\bar{T})+\hat{Q}(t+\bar{T})+S\right),
$$

where $t$ is the age of the forest, and $P$ is the timber price, when the harvest decision is made. ${ }^{15}$ We approximate this payoff at node $\left(P_{i}, t_{j} ; k\right)$ by

$$
\begin{aligned}
\pi_{i, j}^{a, k}= & -\delta+\sum_{n=0}^{\bar{T} / \Delta t-1} e^{-r n \Delta t} \psi_{i+n} \\
& +e^{-r \bar{T}}\left(\left(1-T_{c}\right)\left(P_{i} e^{(r-\kappa) \bar{T}}-h\right) V\left(t_{j}+\bar{T}\right)+Q\left(t_{j}+\bar{T}\right)+S\right) .
\end{aligned}
$$

Similarly, the replanting payoff in equation (4) can be rewritten as

$$
\begin{aligned}
\pi^{r, k}= & -\delta+\int_{0}^{\bar{T}} e^{-r s} \psi(t+s) d s \\
& +e^{-r \bar{T}}\left(\left(1-T_{c}\right)\left(P e^{(r-\kappa) \bar{T}}-h\right) V(t+\bar{T})+\hat{Q}(t+\bar{T})\right)+F(P,-\bar{T} ; k+1),
\end{aligned}
$$

where $k$ signifies the rotation of the forest which is being harvested, and $F(P,-\bar{T} ; k+1)$ is the value of the next planted rotation when the harvest decision is made. It is approximated by

$$
\begin{aligned}
\pi_{i, j}^{r, k}= & -\delta+\sum_{n=0}^{\bar{T} / \Delta t-1} e^{-r n \Delta t} \psi_{i+n} \\
& +e^{-r \bar{T}}\left(\left(1-T_{c}\right)\left(P_{i} e^{(r-\kappa) \bar{T}}-h\right) V\left(t_{j}+\bar{T}\right)+Q\left(t_{j}+\bar{T}\right)\right)+F_{i, 1}^{k+1} .
\end{aligned}
$$

We suppose that the forest owner must abandon forestry after the fifth rotation. ${ }^{16}$ Thus, when $t_{j}=40$ (so that further delay is not allowed), $F_{i, j}^{5}=\pi_{i, j}^{a, 5}$, where $\pi_{i, j}^{a, k}$ is given by equation (B-2). Once the terminal values of the forest in the final rotation are known, the value of the forest may be calculated (for all possible prices and all earlier points in time) through backward induction. In fact, when $t$ is less than 40 years, delay or harvesting followed by abandoning is possible, and the value of the forest is given by

$$
F_{i, j}^{5}=\max \left\{\pi_{i, j}^{d, 5}, \pi_{i, j}^{a, 5}\right\}
$$

\footnotetext{
${ }^{14}$ This grid specification forces the forest owner to make a harvest decision no later than 40 years, but we find that optimal rotations are significantly less than this, so that the restriction does not affect our results.

${ }^{15}$ To simplify the analysis, we assume the abandonment decision is irreversible.

${ }^{16}$ Allowing more than five rotations had no significant impact on our numerical solution.
} 
where $\pi_{i, j}^{d, k}$ is given by equation (B-1).

Similarly, the value of the forest in all prior rotations may be calculated. The only difference is that now we allow the forest owner to replant after harvesting. Thus, when $t_{j}=40$ (and further delay is not allowed), $F_{t}^{i}$ is given by

$$
F_{i, j}^{k}=\max \left\{\pi_{i, j}^{r, k}, \pi_{i, j}^{a, k}\right\}
$$

where $\pi_{i, j}^{r, k}$ is given by equation (B-3). For smaller $t_{j}$, the value of the forest is given by

$$
F_{i, j}^{k}=\max \left\{\pi_{i, j}^{d, k}, \pi_{i, j}^{r, k}, \pi_{i, j}^{a, k}\right\} .
$$

